\title{
Density of universal classes of series-parallel graphs
}

\author{
Jaroslav Nešetřil ${ }^{1 \dagger}$ and Yared Nigussie ${ }^{1 \ddagger}$ \\ ${ }^{1}$ Department of Applied Mathematics \\ Institute for Theoretical Computer Science(ITI) \\ Charles University \\ Malostranské nám.25 \\ 11800 Praha 1 Czech Republic
}

A class of graphs $\mathcal{C}$ ordered by the homomorphism relation is universal if every countable partial order can be embedded in $\mathcal{C}$. It was shown in [1] that the class $\mathcal{C}_{k}$ of $k$-colorable graphs, for any fixed $k \geq 3$, induces a universal partial order. In [4], a surprisingly small subclass of $\mathcal{C}_{3}$ which is a proper subclass of $K_{4}$-minor-free graphs $\left(\mathcal{G} / K_{4}\right)$ is shown to be universal. In another direction, a density result was given in [9], that for each rational number $a / b \in$ $[2,8 / 3] \cup\{3\}$, there is a $K_{4}$-minor-free graph with circular chromatic number equal to $a / b$. In this note we show for each rational number $a / b$ within this interval the class $\mathcal{K}_{a / b}$ of $K_{4}$-minor-free graphs with circular chromatic number $a / b$ is universal if and only if $a / b \neq 2,5 / 2$ or 3 . This shows yet another surprising richness of the $K_{4}$-minor-free class that it contains universal classes as dense as the rational numbers.

Keywords: circular chromatic number, homomorphism, series-parallel graphs, universality

\section{Introduction}

We assume graphs are finite and simple (with no loops and parallel edges). Let $G, G^{\prime}$ be graphs. A homomorphism from $G$ to $G^{\prime}$ is a mapping $f: V(G) \rightarrow V\left(G^{\prime}\right)$ which preserves adjacency. That is, $\{u, v\} \in E(G)$ implies $\{f(u), f(v)\} \in E\left(G^{\prime}\right)$. We write $G \leq G^{\prime}$ if there is a homomorphism from $G$ to $G^{\prime}$. The notation $G<G^{\prime}$ means $G \leq G^{\prime} \not \leq G$, whereas $G \sim G^{\prime}$ means $G \leq G^{\prime} \leq G$. If $G \sim G^{\prime}$, we say $G$ and $G^{\prime}$ are hom-equivalent. The smallest graph $H$ for which $G \sim H$ is called the core of $G$. For finite graphs, the core is uniquely determined up to an isomorphism. It can also be seen that $H$ is an induced subgraph of $G$. This will be denoted by $H \subseteq G$. Let $\mathcal{C}$ and $\mathcal{C}^{\prime}$ be two classes of graphs. We also write $\mathcal{C} \sim \mathcal{C}^{\prime}$ if for each graph $G \in \mathcal{C}$ there exists a $G^{\prime} \in \mathcal{C}^{\prime}$ such that $G \sim G^{\prime}$ and vice versa. See [2] for introduction to graphs and their homomorphisms.

Let $k \geq d \geq 1$ be integers. The circular chromatic number of $G$, written $\chi_{c}(G)$, is the smallest rational $k / d$ such that $G \leq K_{k / d}$, where $K_{k / d}$ is the circular graph with $V\left(K_{k / d}\right)=\{0,1,2, \ldots, k-1\}$ and

\footnotetext{
${ }^{\dagger}$ Supported by a Grant 1M0021620808 of Czech Ministry of Education

‡Partially supported by DIMATIA and 1M0021620808

1365-8050 @ 2005 Discrete Mathematics and Theoretical Computer Science (DMTCS), Nancy, France
} 


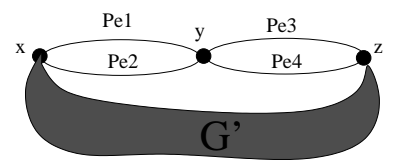

Fig. 1: Unavoidable configuration of $G$ (a minimal counterexample to Lemma 6) with odd girth $2 k+1$ and $l_{e_{1}}+l_{e_{2}}=$ $l_{e_{3}}+l_{e_{4}}=2 k+1$.

$E\left(K_{k / d}\right)=\{\{i, j\}: d \leq|i-j| \leq k-d\}$. Note that when $d=1$ we have the usual vertex coloring of $G$. Let $\mathcal{K}_{a / b}\left\{G \in \mathcal{G} / K_{4}: \chi_{c}(G)=a / b\right\}$. See [10] for some other equivalent definitions. It is trivial to see the following:

Theorem $1 \mathcal{K}_{2} \sim\left\{K_{2}\right\}$.

It is well known that graphs in $\mathcal{G} / K_{4}$ are 3-colorable. Hell and Zhu [3] have shown that triangle-free graphs in $\mathcal{G} / K_{4}$ have circular chromatic number at most $8 / 3$. Hence no graph in $\mathcal{G} / K_{4}$ has circular chromatic number in the interval $(8 / 3,3)$. Hence, we have:

Theorem $2 \mathcal{K}_{3} \sim\left\{C_{3}\right\}$.

The main results of this note are the following two theorems establishing nice dichotomy between universality and homomorphism finiteness of the class $\mathcal{K}_{a / b}$ :

Theorem $3 \mathcal{K}_{5 / 2} \sim\left\{C_{5}\right\}$.

Somewhat surprisingly we show that Theorem 1,2 , and 3 cover all cases when $\mathcal{K}_{a / b}$ is a finite set.

Theorem $4 \mathcal{K}_{a / b}$ is universal if $a / b \in(2,5 / 2) \cup(5 / 2,8 / 3]$.

In section 2 we prove Theorem 3 using a folding lemma. In section 3 we prove Theorem 4.

\section{$2 \mathcal{K}_{5 / 2}$ is equivalent to $\left\{C_{5}\right\}$}

Let $G$ be a graph. A thread in $G$ is a path $P \subseteq G$ such that the two endpoints of $P$ have degree at least 3 and all internal vertices of $P$ are degree 2 in $G$. We shall often use the fact that if $P$ and $P^{\prime}$ are two edge-disjoint paths and if the lengths of $P$ and $P^{\prime}$ have same parity such that $P$ is a thread and has at least equal length as $P^{\prime}$, then there is a homomorphism that maps $P$ to $P^{\prime}$ sending the two ends of $P$ to the two ends of $P^{\prime}$. Such a homomorphism is said to fold $P$ to $P^{\prime}$. Let $G$ be a graph and let $G^{s}$ denote the multi-graph we obtain from $G$ by "smoothing" all degree 2 vertices of $G$. For each edge $e$ of $G^{s}$, let $P_{e}$ denote the thread of $G$ represented by $e$ in $G^{s}$, and let $l_{e}$ denote the length of $P_{e}$.

The following Folding Lemma for $K_{4}$-minor-free graphs is an analogy of the Folding Lemma of Klostermeyer and Zhang [6] for planar graphs. Its proof is easy (see [7]).

Lemma 5 (Edge folding lemma) Let $G \in \mathcal{G} /\left\{K_{4}\right\}$ be of odd girth $2 k+1$ and let e, $e^{\prime}$ be parallel edges in $G^{s}$, with common end vertices $x, y$. If $G$ is not homomorphic to a strictly smaller graph of the same odd girth, then $l_{e}+l_{e^{\prime}}=2 k+1$. Moreover, $P_{e} \cup P_{e^{\prime}}$ is the unique cycle of length $2 k+1$ containing both $x$ and $y$.

For short let $K^{m}$ denote $K_{(7+5 m) /(3+2 m)}$. Recall that $V\left(K^{m}\right)=\{0,1, \ldots, 6+5 m\}$. 
Lemma 6 Let $G \in \mathcal{G} /\left\{K_{4}\right\}$ be of odd girth at least 7 . Then $\chi_{c}(G) \leq(7+5 m) /(3+2 m)<5 / 2$, for some $m<|V(G)| / 2$.

Proof: Let $G \in \mathcal{G} /\left\{K_{4}\right\}$ be a core of odd girth $g \geq 7$. It suffices to show $G \leq K^{m}$ for some $m \geq 0$. Let $\bar{G}^{s}$ be the graph we get by identifying parallel edges of $G^{s}$. Then, $\bar{G}^{s} \in \mathcal{G} / K_{4}$ and so there exists a $y \in V\left(\bar{G}^{s}\right)$ such that the degree $\operatorname{deg}_{\bar{G}^{s}}(y)=2$. Then $3 \leq \operatorname{deg}_{G^{s}}(y) \leq 4$ (here we use a parity argument that, the multiplicity of edges of $G^{s}$ is at most two, as $G$ is a core). By Lemma 5, and assuming $G$ is a minimal counterexample we can get $\operatorname{deg}_{G^{s}}(y)=4$. Hence, a configuration depicted in Figure 1 is unavoidable. Let $G^{\prime}=G-\left(\bigcup_{i=1}^{4} P_{e_{i}}-\{x, z\}\right)$. By induction $G^{\prime} \leq K^{m}$, for some $m \geq 0$. We can assume $f(x)=0$. By investigating a few cases for values of $f(y)$, it is not hard to see $G \leq K^{m+1}$, contrary to assumption (see [7] for detailed proof). $\quad \square$ Proof of Theorem 3: Let $G \in \mathcal{K}_{5 / 2}$ be

of odd girth $g$. Then $G \leq C_{5}$. By Lemma 6, we have $g \leq 5$. By Theorem $2, g>3$. Hence $g=5$ and so $C_{5} \leq G \leq C_{5}$. Hence $G \sim C_{5}$. The converse is obvious since $\chi_{c}\left(C_{5}\right)=5 / 2$.

\section{Universal sets of $\mathcal{G} / K_{4}$ are dense in $(2,5 / 2) \cup(5 / 2,8 / 3]$}

In this section we shall show that we obtain a universal class $\mathcal{K}_{p / q} \subset \mathcal{G} / K_{4}$ for arbitrary $p / q \in(2,5 / 2) \cup$ $(5 / 2,8 / 3]$. We use a graph $G_{p / q}$ with $\chi_{c}(G)=p / q$ as a generator of $\mathcal{K}_{p / q}$. We assume $G_{p / q}$ has the following two properties:

(P1) $G_{p / q}$ is hom-equivalent neither to a cycle nor to a vertex.

(P2) if $G^{\prime} \in \mathcal{G} / K_{4}$ satisfies (P1) and $\chi_{c}\left(G^{\prime}\right)=p / q$, then $\left|V\left(G^{\prime}\right)\right| \geq|V(G)|$.

Lemma 7 Let $G \in \mathcal{G} / K_{4}$ have properties $(P 1)$ and (P2). Then, $G$ is 2-connected. Moreover, $G$ is a core and it is not vertex-transitive.

Proof: Since the circular graph $K_{k / d}$ is a vertex-transitive graph, for all $k, d$, we have $\chi_{c}(G)=$ $\max _{i}\left(\chi_{c}\left(H_{i}\right)\right), 1 \leq i \leq p$, where each $H_{i}$ is a 2-connected component of $G$. Here, (P2) implies that $p=1$ and so $G$ is 2-connected. Next, note that any graph $G \in \mathcal{G} / K_{4}$ is vertex-transitive if and only if $G$ is an odd cycle or $K_{1}$ or $K_{2}$. This is because all other 2-connected graphs in $\mathcal{G} / K_{4}$ have at least one degree-2 vertex and one non-degree-2 vertex. Hence by (P1), $G$ is not vertex-transitive. Moreover, by (P1) the core of $G$ also is not vertex-transitive. By (P2), we deduce $G$ is a core. $\quad \square$ For any rational

number $p / q \in(2,8 / 3$ ], Pan and Zhu have shown in [9] a recursive method of constructing a 2-connected graph $G_{p / q}$ with $\chi_{c}(G)=p / q$. If $p / q \neq(2 k+1) / k$ then $G_{p / q}$ satisfies (P1). If $p / q=(2 k+1) / k$, the graph given in [9] is the cycle $C_{2 k+1}$ which is the natural candidate. Cycles do not satisfy (P1), hence we introduce a graph denoted by $G^{k}$ of odd girth $2 k+3$ as follows: Take a triangle and double each edge to obtain a multi-graph $H$. For $i=0,1,2$, let $\left\{e_{1}^{i}, e_{2}^{i}\right\}$, be the three parallel pairs of edges of $H$. To obtain a thread of length $k+2$, subdivide $e_{1}^{0}$ and $e_{1}^{1}$ each $k+1$ times. Next subdivide $e_{2}^{0}$ and $e_{2}^{1}$ each $k$ times. Finally, subdivide $e_{1}^{3}$ three times and $e_{2}^{3}, 2 k-2$ times to obtain the graph $G^{k}$. We have:

Lemma $8 \chi_{c}\left(G^{k}\right)=(2 k+1) / k$ for all $k \geq 3$.

Proof: It is easy to see that $G^{k} \leq C_{2 k+1}$, and $G^{k} \not \leq C_{2 k+3}$. Hence, we have $(2 k+3) /(k+1)<$ $\chi_{c}\left(G^{k}\right) \leq(2 k+1) / k$. Note that $\operatorname{gcd}(4 k+4,2 k+1)=1$. From basic number theory [8], using what is known as the Farey sequence, we can see that any rational strictly between $(2 k+3) /(k+1)$ and $(2 k+1) / k$ 
has numerator $a \geq 4 k+4$. But then, if $k \geq 3$ the circumference of $G^{k}$ is $4 k+3$. It is well known [10] that the numerator $a$ of a circular chromatic number $a / b$ of a graph $G$ is at most its circumference. We deduce $\chi_{c}\left(G^{k}\right)=(2 k+1) / k$.

Corollary 9 For every rational number $p / q \in(2,5 / 2) \cup(5 / 2,8 / 3]$ there is a graph $G_{p / q}$ satisfying $(P 1)$ and $(P 2)$.

Next we prove that $\mathcal{K}_{p / q}$ inherits universality from the class $\mathcal{P}$ of directed finite paths [5]. We take several isomorphic copies $H_{1}, \ldots, H_{n}$ of a fixed graph $H$, such that $\chi_{c}(H)=p / q$ and construct a 'path-like' structured graph $H^{\prime}$ by identifying a vertex of $H_{i}$ with a vertex of $H_{i+1}$. Then $\chi_{c}\left(H^{\prime}\right)=\chi_{c}(H)$ because the circular graphs are vertex-transitive. We call such a construction $K_{1}$-concatenation. A more precise definition of ' $K_{1}$-concatenation' of a graph $H$ :

Let $P \in \mathcal{P}$ be an oriented path of length $n \geq 1, V(P)=\left\{v_{1}, v_{2}, \ldots, v_{n+1}\right\}$. Then either $v_{i} v_{i+1}$ or $v_{i+1} v_{i} \in E(P)$ (but not both). Let $H$ be a graph and $a, b \in V(H)$. Let $H_{1}, H_{2}, \ldots, H_{n}$ be isomorphic copies of $H$ and let $a_{i}, b_{i}$ be the vertices of $H_{i}$ corresponding to $a$ and $b$. The $K_{1}$-concatenation of $H$ by $P$ is a graph $P *(H, a, b)$ constructed as follows: For $i=1, \ldots, n$, if $v_{i} v_{i+1} \in E(P)$, choose $b_{i}$ (otherwise choose $a_{i}$ ). Then identify every chosen vertex of $H_{i}$ with the unchosen vertex of $H_{i+1}$. To make the construction non-trivial, we choose $a$ and $b$ so that there is no automorphism sending $a$ to $b$ or $b$ to $a$. If $H$ satisfies (P1), then we know there exists such a pair.

Lemma 10 Let $G_{p / q} \in \mathcal{G} / K_{4}$ satisfy $(P 1),(P 2)$. Then, $\mathcal{K}_{p / q}$ is universal.

Proof: Since the class of oriented paths $\mathcal{P}$ is universal we show for every $P, P^{\prime} \in \mathcal{P}$, we have $P \leq P^{\prime}$ if and only if $P *\left(G_{p / q}, a, b\right) \leq P^{\prime} *\left(G_{p / q}, a, b\right)$. This proves the lemma.

The forward implication is straightforward. To prove the reverse implication, let $P, P^{\prime} \in \mathcal{P}$ of length $n$ and $n^{\prime}$ such that $P$ is a core and suppose there exists a homomorphism $f: P *\left(G_{p / q}, a, b\right) \rightarrow P^{\prime} *$ $\left(G_{p / q}, a, b\right)$. Assume further that $P$ is not an edge, since this case is trivial, and without loss of generality, assume that the first edge of $P$ is directed forward. Let $H_{1}, \ldots, H_{n}$ and $H_{1}^{\prime}, \ldots, H_{n^{\prime}}^{\prime}$ be isomorphic copies of $G_{p / q}$. First we show that $\left.f\right|_{H_{i}}$ is induced by an automorphism of $G_{p / q}$ for each $i$. Suppose not. Then the image $f\left(H_{i}\right)$ is connected. If $f\left(H_{i}\right)$ is 2-connected then, it is isomorphic to $G_{p / q}$, since $G_{p / q}$ is a core. Suppose $f\left(H_{i}\right)$ is not 2-connected. Then each 2-connected component $F$ of $f\left(H_{i}\right)$ is a proper subgraph of $G_{p / q}$. By (P2), we have $\chi_{c}(F)<p / q$. Then, $H \not \leq f\left(H_{i}\right)$, a contradiction. Hence $\left.f\right|_{H_{i}}$ is induced by an automorphism of $G_{p / q}$.

Now we claim a stronger assertion that for any $i, f\left(a_{i}\right)=a_{j}^{\prime}$ and $f\left(b_{i}\right)=b_{j}^{\prime}$, for some $j, 1 \leq j \leq m$. let $H_{j}^{\prime}=f\left(H_{1}\right)$. Since $\left.f\right|_{H_{1}}$ is an automorphism, $f\left(b_{1}\right)$ must be in the same automorphism class of $b_{j}^{\prime}$. Suppose that $f\left(b_{1}\right) \neq b_{j}^{\prime}$, then $f\left(b_{1}\right)$ is not a cut-vertex of $P^{\prime} *\left(G_{p / q}, a, b\right)$. As $b_{1}$ is a cut-vertex of $P *\left(G_{p / q}, a, b\right)$, it is identified with either $a_{2}$ or $b_{2}$. If it were identified with $a_{2}$, then $\left.f\right|_{H_{2}}$ would be an automorphism of $G_{p / q}$ such that $f\left(a_{2}\right)=b_{j}^{\prime}$, contrary to the choice of $a, b$ in $V\left(G_{p / q}\right)$. Hence $b_{1}$ is identified with $b_{2}$. Similarly, we get $a_{2}$ is identified with $a_{3}$, and $b_{3}$ with $b_{4}$ and so on. This implies $P$ is a 'zig-zag' which is hom-equivalent to an edge, contrary to $P$ being a core. So $f\left(b_{1}\right)=b_{j}^{\prime}$. The claim follows by induction on the length of $P$.

We define a homomorphism $g: V(P) \rightarrow V\left(P^{\prime}\right)$ so that if $f\left(a_{i}\right)=a_{j}^{\prime}$ then $g\left(v_{i}\right)=v_{j}^{\prime}$ and similarly for $b_{i}$. By our construction $g$ preserves the adjacency condition and so $P \leq P^{\prime}$. $\square$ Proof of Theorem 4: Let $a / b \in(2,5 / 2) \cup(5 / 2,8 / 3]$. By Lemma 9, there is a graph $G_{a / b}$ with properties (P1),(P2). By Lemma $10, \mathcal{K}_{a / b}$ is universal. This concludes our result. 


\section{References}

[1] Z. Hedrlín, On universal partly ordered sets and classes, J. Algebra 11(1969),503-509.

[2] P.Hell, J. Nešetřil, Graphs and Homomorphisms, Oxford University Press, 2004.

[3] P.Hell, X. Zhu, The circular chromatic number of series-parallel graphs, J. Graph Theory, $33(2000), 14-24$.

[4] J. Hubička, J. Nešetřil, Universal Partial Order Represented by Means of Trees and Other Simple Graphs, (to appear in European J. Comb.)

[5] J. Hubička, J. Nešetřil , Finite Paths are Universal, ITI Series 2003-129, Charles University, 2003 (to appear in Order)

[6] W. Klostermeyer, C.Q. Zhang, $(2+\epsilon)$-coloring of planar graphs with prescribed girth, J. Graph Theory, 33(2000) (2):109-119.

[7] J. Nešetřil, Y. Nigussie, Density of universal classes of series-parallel graphs (KAM Series 2004-717)

[8] I. Niven, H. Zuckerman and H. Montgomery, An introduction to the Theory of Numbers, Hojn Wiley \& Sons, Inc., Fifth Edition, 1991.

[9] Z. Pan, X. Zhu, Density of the circular chromatic number series-parallel graphs, J. Graph Theory, 46(2004), 57-68.

[10] X. Zhu, Circular Chromatic Number, a survey. Discrete Mathematics, Vol.229 (1-3)(2001), 371-410. 
